\title{
Applications of Pseudospark Produced Electron Beams in Millimetre Wave Radiation Sources
}

\author{
A.W. Cross ${ }^{1}$, W. He ${ }^{1}$, L. Zhang ${ }^{1}$, H. Yin ${ }^{1}$, D. Bowes ${ }^{1}$, K. Ronald ${ }^{1}$, A.D. R. Phelps ${ }^{1}$, Y. Yin ${ }^{2}$, G. Liu ${ }^{2}$, \\ D. $\mathrm{Li}^{3}$ and $\mathrm{X} . \mathrm{Chen}^{3}$ \\ ${ }^{1}$ Department of Physics, SUPA, University of Strathclyde, Glasgow, G4 0NG, UK \\ ${ }^{2}$ School of Physical Electronics, University of Electronic Science \& Technology of China, Chengdu, China \\ ${ }^{3}$ School of Electronic Engineering \& Computer Science, Queen Mary, University of London, London E1 4NS, UK
}

\begin{abstract}
Pseudospark (PS) electron beams of outstanding performance have been studied recently with their application to a demanding field of millimeter-wave and terahertz radiation generation. To this end, the PS discharge process itself has been studied and millimeter wave sources which utilize a PS sourced electron beam in different beam-wave interaction structures have been designed and modelled using the particle-in-cell code MAGIC. The experimental demonstration of the PS-sourced electron beams of sub-millimeter diameter and the coherent millimeter wave radiation generated from PS sourced electron beams in different beam-wave interaction structures will be presented.
\end{abstract}

Keywords-Pseudospark discharge, beam-wave interaction, millimeter wave radiation.

\section{INTRODUCTION}

Radiation sources in the terahertz region (0.1-10 THz) have received increasing interest in recent years because of the demand in the fields of plasma diagnosis, material science, radiotherapy, spectroscopy and advanced communications. In vacuum electronics electron beam sources have been always crucial for radiation generation, the pseudospark (PS) discharge has attracted a lot of interest recently as a promising source of high quality, high intensity electron beam pulses.

\section{PSEUDOSPARK DISCHARGE}

A PS is an axially symmetric, self-sustained, transient, low pressure (typically 50-500 mTorr) gas discharge in a hollow cathode/planar anode configuration, which operates on the low pressure side of the hollow cathode analog to the Paschen curve [1-3]. A useful property of this type of discharge is the formation of an electron beam during the breakdown process. During a PS discharge, low temperature plasma is formed as a copious source of electrons and can be regarded as a low work function surface that facilitates electron extraction [4-8]. Because of the special geometry and discharge mechanism, the electron beam from a PS discharge can propagate without an external magnetic guiding field due to the existence of an ion-focusing channel. The ion-focusing channel is formed due to the background gas ionization by the front of the electron beam itself. For generation of high frequency radiation in millimeter wave and sub-millimetre wave region this beam is ideal due to its small beam size, compactness and long lifetime. This paper presents experimental results of the production of PS-sourced electron beams of sub-millimeter diameter.

At Strathclyde University the PS discharge and its potential applications in high frequency radiation generation have been investigated both experimentally and numerically. Fig. 1 is a typical PS-sourced electron beam experimental setup in which a 4-gap PS discharge chamber is combined with a collimator with micron aperture of different diameters of 500 $\mu \mathrm{m}, 200 \mu \mathrm{m}, 100 \mu \mathrm{m}$ and $70 \mu \mathrm{m}$ for micro beam extractions. A phosphor scintillator disc was introduced into the beam drift tube to acquire the beam image. Fig. 2 shows a typical single gap PS discharge structure and its simulation compared with discharge experiments. From the simulation, it can be seen that the plasma formed at a trigger electrode expands inside the hollow cathode region and then enters into the gap region to complete a discharge cycle, during which electrons are collected at the anode aperture as shown in the middle of Fig. 2. The evolution of both the discharge voltage and collected beam current agrees well with experimental measurements as shown at the bottom of Fig. 2.

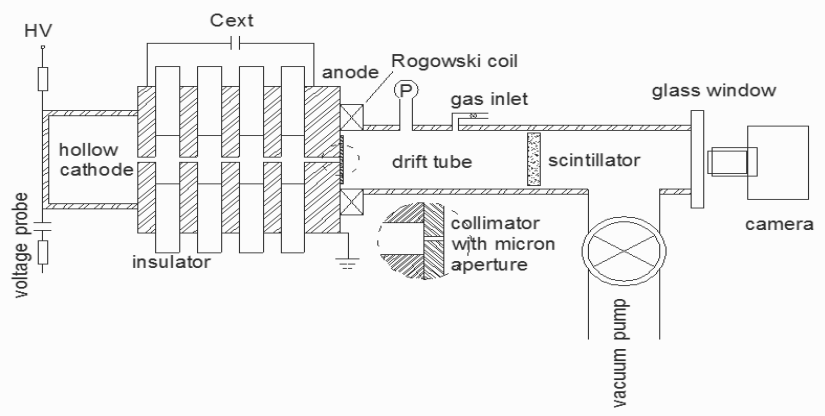

Fig. 1 Typical pseudospark-sourced electron beam electron experimental setup of a 4-gap pseudospark system. 

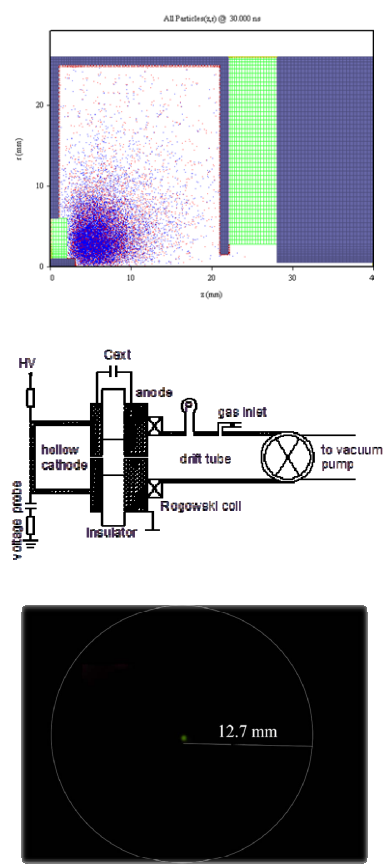

$500 \mu \mathrm{m}$ image
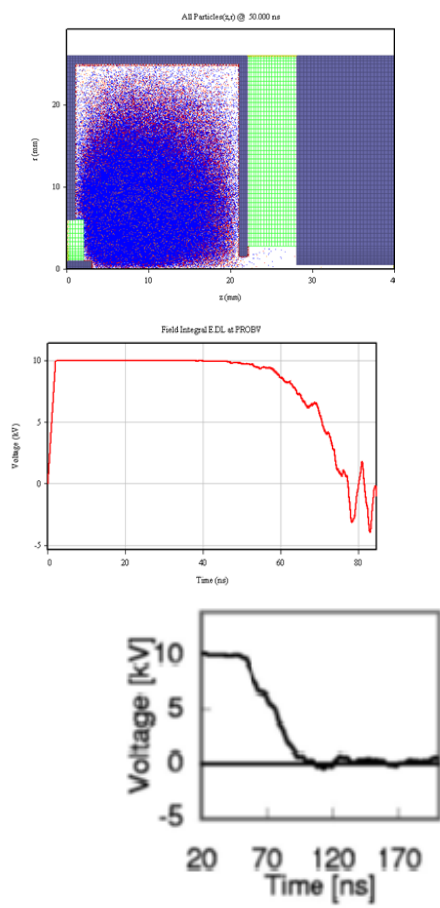

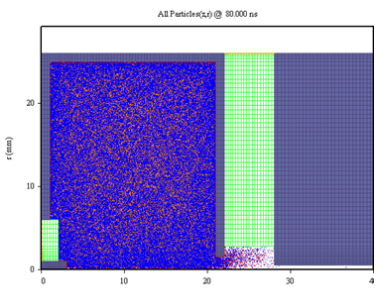

$z(\min )$

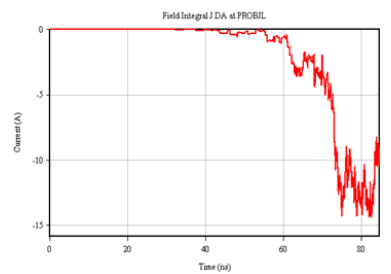

150

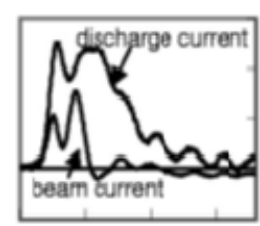

$100 \bar{\Xi}$

50 है

0 동

$-50$

Time [ns]

Fig. 2 Typical single gap PS discharge structure (middle and right) and its simulation (top and middle, centre and right graphs) compared with discharge experiment (bottom) of scintillator image of beam cross section, discharge voltage \& current and beam current.

\section{DIFFERENT TYPES OF INTERACTION CIRCUITS}

Fig. 3 shows the schematic experimental setup of a 4-gap PS sourced electron beam that in the first instance was used to drive a backward wave oscillator (BWO) but which can also be used to drive different beam-wave interactions such as a micro-klystron and an extended interaction oscillator (EIO) in W-band (75 to 110$) \mathrm{GHz}$ and G-band (140 to 220) GHz.

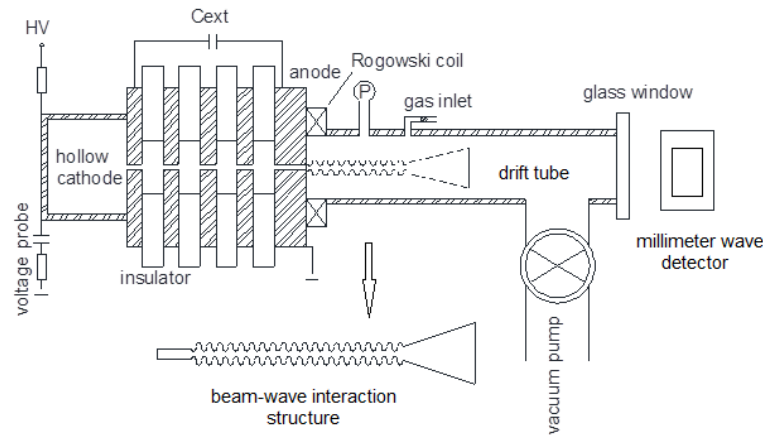

Fig. 3 Schematic experimental setup of a 4-gap pseudospark sourced electron beam used to drive a BWO.

\section{A. W-band $B W O$}

A slow wave structure (SWS) was designed for the Wband BWO, Fig 4(a). The operating mode is the $\mathrm{TM}_{01}$. The beam-wave interaction was simulated by MAGIC and the interaction frequency is about $70 \mathrm{GHz}$. The beam trajectories and the beam energy is shown in Fig. 4(b). Fig. 4(a) shows a photograph of the manufactured $\mathrm{W}$-band $\mathrm{BWO}$ structure and Fig. 5 shows the measured voltage, current and mm-waves.

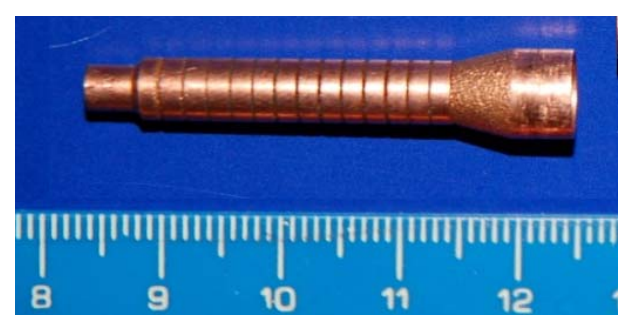

(a)
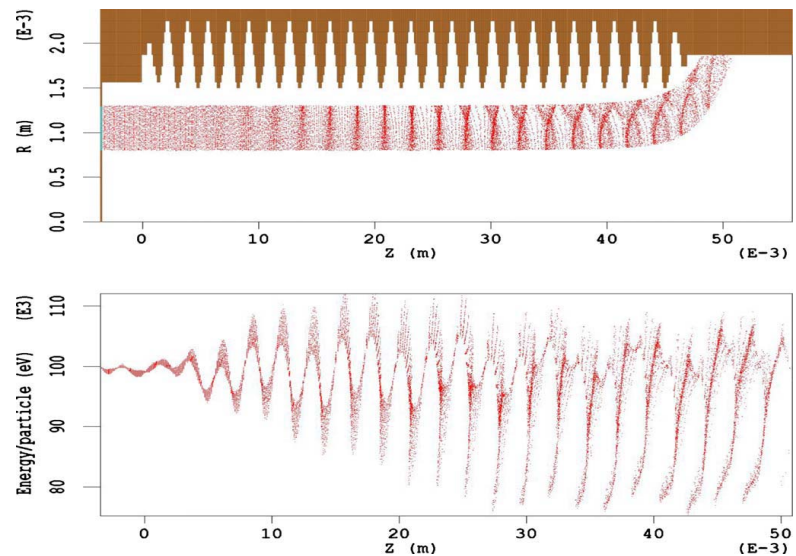

(b)

Fig.4 (a) Manufactured W-band BWO structure, (b) MAGIC simulation of the electron trajectories when interacting with the corrugated backward traveling wave structure (top ) with the beam electron bunches and beam electron energy. 


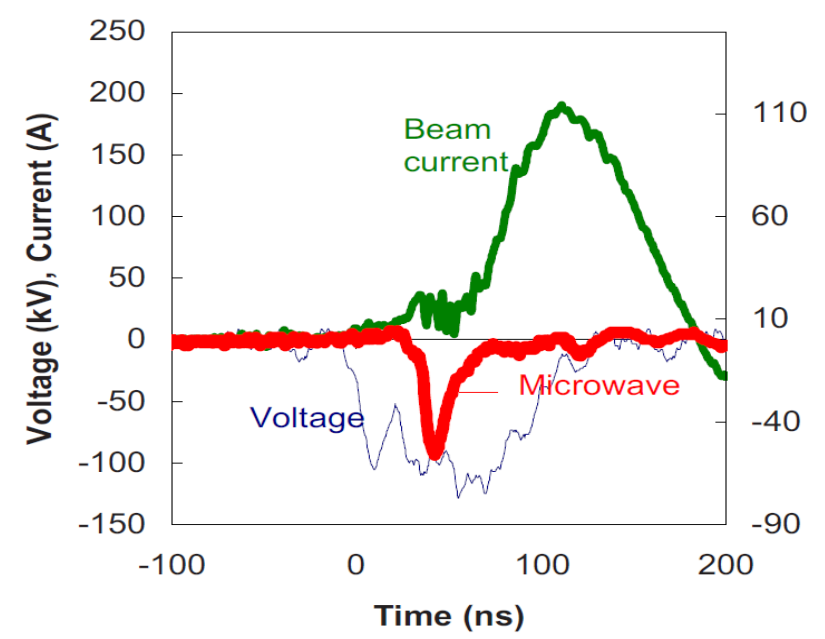

Fig.5 The beam voltage, beam current and microwave pulse in the experiment.

\section{B. W-band klystron}

A three-cavity $\mathrm{W}$-band klystron was designed to operate at beam voltage of $8 \mathrm{kV}$ and beam current of $15 \mathrm{~mA}$. The operating frequency is $96 \mathrm{GHz}$ and the gain is $45 \mathrm{~dB}$ in the MAGIC simulation. Fig. 6 and Fig. 7 show the 3D models of the interaction cavities and the experimental setup.

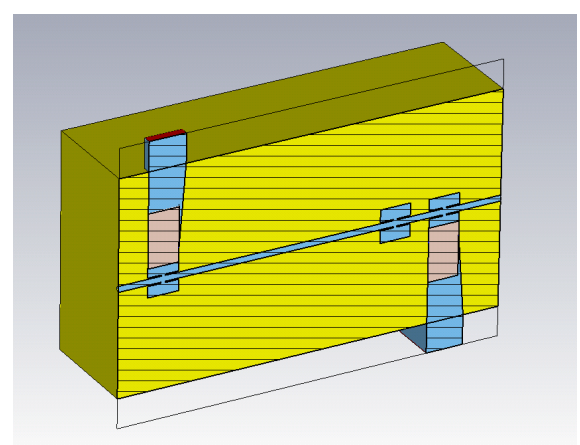

Fig. $63 \mathrm{D}$ model of the designed three-cavity W-band klystron.

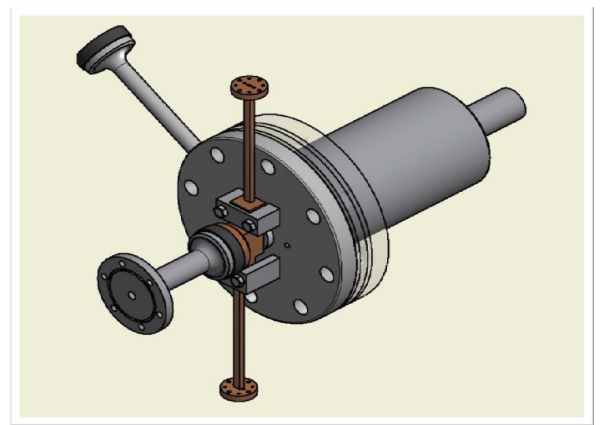

Fig. 7 Experimental setup of the W-band klystron.

\section{W-band EIO}

An EIO structure has been designed to operate with the pseudospark generated electron beam. The operating voltage is $30.5 \mathrm{kV}$ and the output frequency is $94 \mathrm{GHz}$. The $3 \mathrm{D}$ EIO structure is shown as Fig. 8(a) and was machined by wirecutting technique, as shown in Fig. 8(b). It was then cold tested by using a vector network analyzer (VNA) and its frequency was found to be $93.8 \mathrm{GHz}$. Fig. 9 shows a photograph of the experimental setup for the W-band EIO. Experimental results with a pseudospark electron beam will be presented.

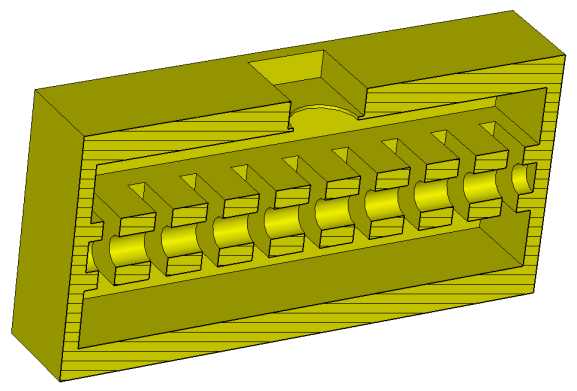

(a)

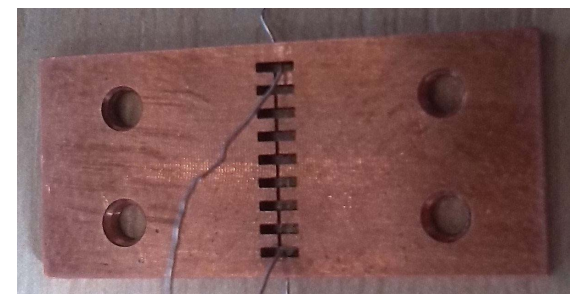

(b)

Fig.8 3D model of the W-band EIO and the machined inteaction circuit.

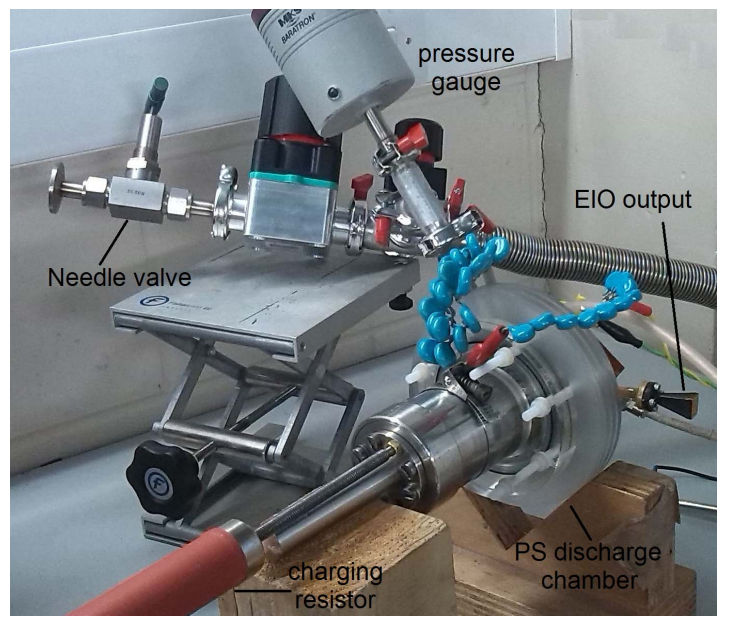

Fig.9 Experimental setup for the W-band EIO.

\section{G-band $B W O$}

A corrugated SWS has been designed to operate at G-band to enable the generation of microwave radiation with swept frequencies. The operating mode is the $\mathrm{TM}_{01}$ and it was able to interact with electron beam from $42 \mathrm{kV}$ to $25 \mathrm{kV}$. The beamwave interaction was simulated using the PIC code MAGIC in 2D. Fig. 10 shows the predicted frequency spectrum, which covers the frequency range of $186-200 \mathrm{GHz}$. To verify the 
simulation, a SWS has been machined and is shown in Fig. 11. Further experiments will be carried out using this structure.

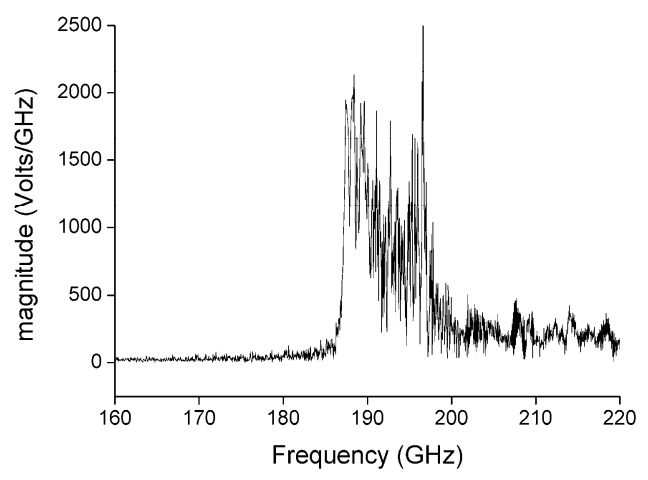

Fig.10 Frequency spectrum as predicted using 2D PIC code MAGIC.

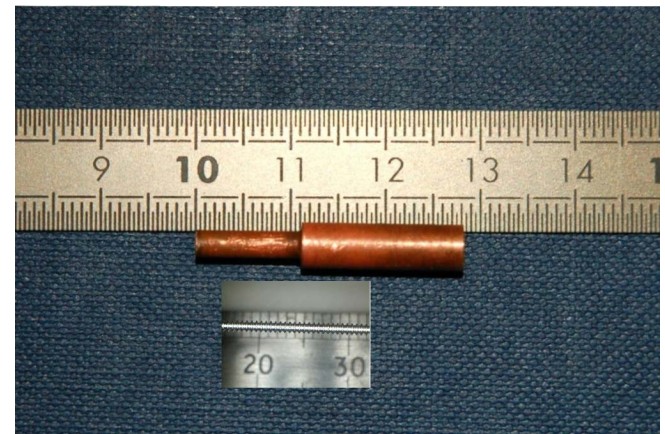

Fig.11 Photographs of G-band BWO structure (top) and its former (bottom).

\section{E. G-band EIO}

A SWS with double staggered metallic rod array (DSMRA) was studied to interact with a sheet electron beam, as shown in Fig. 12. The simulations show with a $800 \mu \mathrm{m} \times$ $100 \mu \mathrm{m} 30 \mathrm{kV}, 200 \mathrm{~mA}$ sheet beam, an output power of 133.1 $\mathrm{W}$ can be achieved at $210.4 \mathrm{GHz}$. The interaction efficiency is about $2.2 \%$. In the experiment, a collimator with rectangular aperture will be applied to the pseudospark anode to obtain a sheet electron beam. Detail measurement of the frequencies and power of the radiation will be carried out.

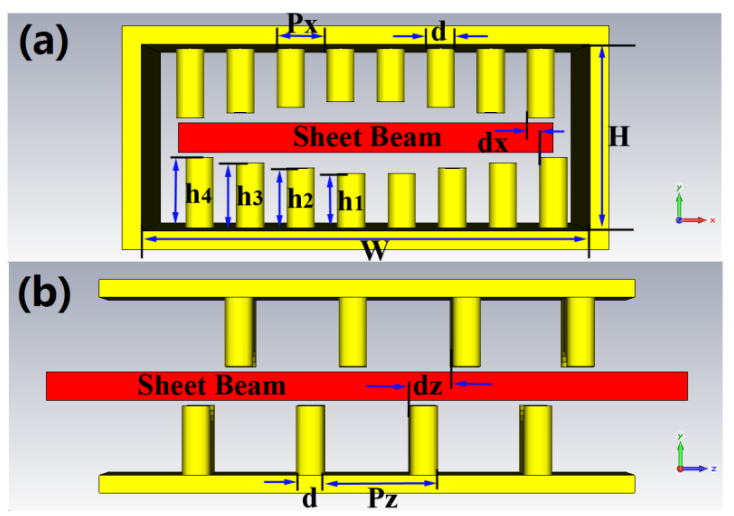

Fig.12 DSMRA SWS structural model: (a) front view and (b) side view.

\section{CONCLUSION}

Various beam-wave interaction circuits covered a wide range of frequencies in W-band and G-band have been studied to investigate the potential applications of the speudospark generated electron beams.

\section{Acknowledgment}

This work was supported by the Engineering and Physical Sciences Research Council (EPSRC) U.K. under Research Grant EP/K029746/1.

\section{References}

[1] J. Christiansen and C. Schultheiss, "Production of high-current particle beams by low-pressure spark discharges", Zeitshrift Fur Physik AHadrons and Nuclei, 290, No. 1, pp35-41, (1979)

[2] M. A. Gunderson and G. Schaefer, "The effect of pendel electrons on breakdown and sustainment of a hollow-cathode discharge", NATO Advance Science Institute Series, Series. B, New York: Plenum (1990)

[3] K. Frank and J. Christiansen, "The fundamentals of the psuedospark and its applications" IEEE Trans. Plasma Sci., 17, 748 (1989).

[4] H. Yin H., A.W. Cross, W. He, A.D.R. Phelps and K. Ronald, "Pseudospark Experiments: Cherenkov Interaction and Electron beam Post-acceleration", 2nd Special Edition of the IEEE Transactions on Plasma Science on Pseudospark Physics and Applications, PS-32, pp233-239, 2004.

[5] H. Yin, A. W. Cross, W. He, A. D. R. Phelps, K. Ronald, D. Bowes, C.W. Roberson "Millimeter wave generation from a pseudosparksourced electron beam".,Phys. Plasmas 16 (2009).

[6] G. Liu, W. He, A.W. Cross, H. Yin and D. Bowes, "Simulation of a Gband sheet beam backward wave oscillator with double staggered metallic rod array", J. Phys. D: Appl. Phys., 46, 345102 (2013).

[7] D. Bowes, H. Yin, W. He, L. Zhang, A.W. Cross, K. Ronald, A.D.R. Phelps, D. Chen, P. Zhang, X. Chen and D. Li, "X-ray Emission as a Diagnostic from Pseudospark-Sourced Electron Beams", Nuclear Inst. and Methods in Physics Research, B 235, pp74-77, (2014).

[8] D. Bowes, H. Yin, W. He, A.W. Cross, K. Ronald, A.D.R. Phelps, D. Chen, P. Zhang, X. Chen and D. Li, "Visualization of a PseudosparkSourced Electron Beam", IEEE Transaction on Plasma Science, 42, 10, pp2826-2827, (2014) 